\title{
Regulatory effects of Ningdong granule on dopaminergic and serotonergic neurotransmission in a rat model of Tourette syndrome assessed by PET
}

\author{
YUAN WANG and ANYUAN LI \\ Department of Traditional Chinese Medicine, Shandong Provincial Hospital Affiliated to Shandong University, \\ Jinan, Shandong 250021, P.R. China
}

Received September 4, 2018; Accepted March 20, 2019

DOI: $10.3892 / \mathrm{mmr} .2019 .10243$

\begin{abstract}
Dysfunctions in dopamine (DA) and serotonin (5-HT) metabolism have been widely implicated in Tourette syndrome (TS); however, the exact nature of these dysfunctions remains unclear. The objective of the present study was to investigate the variation in DA and 5-HT metabolism in a rat model of TS, and to evaluate the therapeutic effect of Ningdong granule (NDG), a traditional Chinese medicine (TCM) preparation used specifically for the treatment of TS. Rats were treated with 3,3'-iminodipropionitrile for 7 days to induce the model of TS, and were then intragastrically administered NDG each day. After 8 weeks of treatment, micro-positron emission tomography was used to measure the binding of DA $\mathrm{D}_{2}$ receptors $\left(\mathrm{D}_{2} \mathrm{Rs}\right)$, DA transporters (DATs), $5-\mathrm{HT}_{2 \mathrm{~A}}$ receptors $\left(5-\mathrm{HT}_{2 \mathrm{~A}} \mathrm{Rs}\right)$ and $5-\mathrm{HT}$ transporters (SERTs) in brain regions of interest. The results indicated that NDG could significantly reduce the typical characteristics of TS in the rat model. Decreased $\mathrm{D}_{2} \mathrm{R}$ binding and increased DAT binding were detected in the striatum compared with the binding activities in untreated rats. The density of $5-\mathrm{HT}_{2 \mathrm{~A}} \mathrm{R}$ was also significantly increased in the striatum following NDG treatment; however, SERT levels were decreased in certain brain regions, including the striatum, cortex, nucleus accumbens and amygdala. Taken together, the current results demonstrated that NDG may be effective in treating patients with TS.
\end{abstract}

Correspondence to: Professor Anyuan Li, Department of Traditional Chinese Medicine, Shandong Provincial Hospital Affiliated to Shandong University, 324 Jingwuweiqi Road, Jinan, Shandong 250021, P.R. China

E-mail: professorli@126.com

Key words: Tourette syndrome, traditional Chinese medicine, dopamine, serotonin, micro-positron emission tomography

\section{Introduction}

Tourette syndrome (TS) is a disorder that is characterized by tics. Tics are best described as normally voluntary movements that are instead made automatically, and are therefore not under the control of the individual. It is estimated that $0.3-0.9 \%$ of children worldwide have TS (1). TS affects males more frequently than females and is often associated with attention deficit hyperactivity disorder (ADHD) and obsessive-compulsive disorder (OCD). There is frequently an urge that precedes the tic, sometimes in the form of a specific sensory feeling (a sensory tic) (2). Patients state that they perform the tic in order to reduce the urge, although shortly after the tic manifests, the urge recurs.

The precise aetiological and pathophysiological mechanisms underlying TS with or without co-morbid conditions remain unknown. Nevertheless, the symptoms can be aggravated by psychosocial stress, anxiety, infection, emotional tension and/or fatigue. There is evidence to show that TS is passed down through families (3), although the genetic component or combination of components has not yet been identified $(4,5)$. Clinical, neuropathological and neuroimaging studies, as well as autopsy, have suggested that abnormalities of the basal ganglia and in neurotransmitter function are associated with TS $(6,7)$. Of the known neurotransmitters, catecholamines and serotonin (5-HT) may play significant roles in the development and occurrence of tics (8-10). Treatments for TS are variable and include pharmacological and behavioural treatments, surgery and deep brain stimulation, among other methods (11). Current commonly used anti-tic drugs include $\alpha$-adrenergic receptor agonists, typically neuroleptics and atypical neuroleptics. However, there is no method that can fully cure TS at present. Due to the high efficacy of dopamine (DA) D2 receptor $\left(\mathrm{D}_{2}-\mathrm{R}\right)$ antagonists, including haloperidol (Hal), abnormalities in DA metabolism have been proposed to be associated with TS $(9,12)$. Increasingly, nuclear imaging studies and neurochemical assays of post-mortem brain tissues isolated from patients with TS have demonstrated dysfunction in the dopaminergic system $(13,14)$. Meanwhile, the 5-HT system has also been implicated as having an important role in the pathophysiology of TS (15). Individuals with OCD often 
respond to 5-HT re-uptake inhibitors (16), and it has been reported that dual-acting serotonergic/dopaminergic agents, including risperidone, help to alleviate the symptoms of TS and OCD (17). Consequently, it may be speculated that 5-HT contributes to the symptoms of TS.

3,3'-Iminodipropionitrile (IDPN) has frequently been used to develop animal models of tics, as it can cause persistent behaviour syndromes that include head shaking, random circling, hyperactivity and increased acoustic startle responses (18). These stereotyped behaviours are similar to the symptoms of TS, and are attributed to variations in processes involving DA, 5-HT and certain other peptides, including norepinephrine (19). Traditional Chinese Medicine (TCM) is a practice that has been used for thousands of years due to its apparent effectiveness and infrequent side effects. Ningdong granule (NDG), a compound preparation used in TCM, is applied to alleviate tics, for which it has been indicated to be effective (20-22). In the present study, the focus was on the DA and 5-HT systems in order to evaluate the anti-tic function of NDG in an animal model. First, IDPN was used to develop a rat model of TS; subsequently, the rats were divided into four groups, treated with saline, Hal or NDG. After 8 weeks, micro-positron emission tomography (PET) was used to evaluate the binding of $\mathrm{D}_{2} \mathrm{Rs}$, DA transporters (DATs), $5-\mathrm{HT}_{2 \mathrm{~A}}$ receptors $\left(5-\mathrm{HT}_{2 \mathrm{~A}} \mathrm{Rs}\right)$ and 5-HT transporters (SERTs) in brain regions of interest (ROIs).

\section{Materials and methods}

Drugs and reagents. IDPN was purchased from Sigma-Aldrich (Merck KGaA), Hal was from Shanghai Pharmaceutical Group Co., Ltd., and isotopic tracers (radiochemical purity $>99 \%$ ) were synthesized and provided by the Jiangsu Institute of Atomic Medicine (Wuxi, China).

Preparation of NDG. The NDG formula includes 10 different Chinese medicinal herbs (Table I). All of these formulations were provided and prepared by Tianjiang Medicine Co., Ltd. After being dried, the ingredients were mixed at the proportions listed in Table I and then macerated for $1 \mathrm{~h}$ at room temperature in distilled water $(1,000 \mathrm{ml})$. Subsequently, the mixture was decocted twice for $1 \mathrm{~h}$ each time at $100^{\circ} \mathrm{C}$. The filtrates were mixed and condensed and then dried in a vacuum drier at $60^{\circ} \mathrm{C}$ until they became granular. The resulting granules were stored at $4^{\circ} \mathrm{C}$.

Experimental animals. All experimental procedures were performed in compliance with the relevant guidelines and regulations of the American Physiological Society, and the protocols were approved by the medical ethics committee of The Provincial Hospital Affiliated to Shandong University.

A total of 24 male Wistar rats (5 weeks old, $150 \pm 10 \mathrm{~g}$ ) were purchased from Shanghai Laboratory Animal Co., Ltd.; the rats were divided into five cages and housed in an air-conditioned animal room under a $12 \mathrm{~h}$ light/dark cycle (lights on at 06:00 a.m. and off at 6:00 p.m.). Animals were provided access to water and food ad libitum, were maintained at a constant temperature of $22 \pm 2^{\circ} \mathrm{C}$ and a humidity of $50 \pm 10 \%$, and were given a 1 -week adaptation period. After this period, the rats were randomly divided into a control group $(n=6)$ and an experimental group $(n=18)$. Animals in the control group were intraperitoneally (i.p.) injected with normal saline (NS; $0.9 \%, 5 \mathrm{ml} / \mathrm{kg}$ ); animals in the experimental group were injected with IDPN (150 mg/kg, i.p.). Injections for both groups were administered once a day for 7 consecutive days. The experimental rats were further randomly assigned to three groups: An IDPN+NS group $(n=6)$, an IDPN+Hal group $(n=6)$ and an IDPN+NDG group $(n=6)$. In these groups, following the i.p. injection of IDPN, rats were treated by intragastric administration of NS $(0.9 \%)$ at $10 \mathrm{ml} / \mathrm{kg}$ (IDPN+NS group), Hal at $1.0 \mathrm{mg} / \mathrm{kg}$ (IDPN+Hal group) or NDG at $22 \mathrm{~g} / \mathrm{kg}$ (IDPN+NDG group; 6.25-fold greater than a clinical dose) once a day for 8 weeks. The behaviours of the rats were observed by researchers who were familiar with stereotypical behaviours, but blinded to the experimental group of each rat, once every 7 days after IDPN and drug administration. Each animal was observed for $1 \mathrm{~min}$ out of every $5 \mathrm{~min}$ over a total of 6 periods. Episodes that were in accordance with specific behavioural categories (Table II) received the corresponding score, and an average score was calculated on the basis of the results from two observers to determine an objective indicator of behavioural changes.

MicroPET scans. In preparation for the scans, the rats were anaesthetized with $1-1.5 \%$ isoflurane and then injected via the tail vein with $150-250 \mu \mathrm{l}(250 \pm 50 \mu \mathrm{Ci}){ }^{18} \mathrm{~F}$-fallypride (a compound that binds predominantly to $\mathrm{D}_{2}$-Rs), ${ }^{18} \mathrm{~F}$-FECNT (binds to DATs), ${ }^{18} \mathrm{~F}$-altanserin (binds to $5-\mathrm{HT}_{2 \mathrm{~A}} \mathrm{Rs}$ ) or ${ }^{18}$ F-FPBM (binds to SERTs). After $10 \mathrm{~min}$ to allow for radiotracer uptake, the rats were positioned on a bed in pairs, with the two rats lying in prone positions, and then placed in a microPET scanner (Focus 220 scanner; Siemens AG). Scans lasted $10 \mathrm{~min}$. Images were reconstructed using an iterative three-dimensional ordered-subset expectation maximization algorithm with an image matrix of $128 \times 128 \times 159$, resulting in a pixel size of $0.77 \mathrm{~mm}$ and a slice thickness of $0.78 \mathrm{~mm}$. The anatomical ROIs, namely the striatum, cortex, nucleus accumbens, putamen and amygdala were delineated with the PMOD software package (version 3.8; PMOD Technologies LLC), and the uptake of the radiotracers [standard uptake ratio (SUV)] was calculated using the following formulae: SUV = Radioactive material ingestion in the area of interest $(\mu \mathrm{Ci} / \mathrm{g}) /$ Total injection dose $(\mu \mathrm{Ci}) /$ Weight $(\mathrm{g})$.

Statistical analysis. The results are expressed as the mean \pm SEM. Statistical differences between groups were determined by one-way analysis of variance. The Least Significant Difference test was used for the comparison of parameters between different groups. All data were analysed with the SPSS statistical software package (version 17.0; SPSS Inc.), and $\mathrm{P}<0.05$ was considered to indicate a statistically significant difference.

\section{Results}

Behavioural evaluation. As indicated in Fig. 1, IDPN caused abnormal, stereotypical behaviours in rats. After 8 weeks of treatment, these abnormal behaviours were significantly reduced in the IDPN plus NDG and IDPN plus Hal groups 
Table I. Composition and active compounds of Ningdong granule.

\begin{tabular}{lccc}
\hline Components & Voucher specimens no. & Part used & Amount used (g) \\
\hline Gastrodia elata Blume & 1404620 & Root & 9 \\
Uncaria rhynchophylla (Miq.) Jacks & 1404631 & Ramulus & 15 \\
Buthus martensii Karsch & 1404676 & Dried body & 3 \\
Scolopendra subspinipes mutilans L. Koch & 1404674 & Dried body & Single band \\
Fossil fragments & 1405688 & Skeletal fossils & 30 \\
Radix Paeoniae Alba & 1406089 & Root & 20 \\
Dwarf lilyturf tuber & 1407051 & Root & 10 \\
Dried human placenta & 1311055 & Dried placenta & 3 \\
Codonopsis pilosula & 1403701 & Rhizome & 10 \\
Glycyrrhiza uralensis Fisch & 1403710 & Rhizome & 3 \\
\hline
\end{tabular}

Table II. Scales for stereotypical behaviours.

\begin{tabular}{ll}
\hline Score & \multicolumn{1}{c}{ Stereotypical behaviours } \\
\hline 0 & $\begin{array}{l}\text { Asleep, resting in place or normal activity in place } \\
\text { Increased sniffing and head raising }\end{array}$ \\
2 & $\begin{array}{l}\text { Discontinuous increased sniffing with body raising } \\
\text { Discontinuous increased sniffing and/or licking with } \\
\text { head and body raising primarily in one place, with } \\
\text { occasional rapid bursts of locomotor activity (2-5 } \\
\text { steps) }\end{array}$ \\
Continuous sniffing, biting and head bobbing and \\
repetitive body raising/wall climbing in place \\
Continuous sniffing, biting, licking and head bobbing, \\
and continuous body raising/wall climbing wherein \\
forepaws do not touch the cage floor
\end{tabular}

compared with the IDPN plus NS group $(\mathrm{P}<0.05)$. There was no statistically significant difference in the behaviours of rats between the NDG and Hal groups.

\section{MicroPET imaging.}

$D_{2} R$. In the striatum, IDPN increased the level of $\mathrm{D}_{2} \mathrm{R}$ binding compared with that in the control group $(\mathrm{P}<0.05)$. Following treatment, NDG or Hal significantly reduced the upregulation in striatal $\mathrm{D}_{2} \mathrm{R}$ content $(\mathrm{P}<0.01)$. No significant difference was observed between the NDG and Hal groups (Fig. 2).

$D A T$. The DAT content in the striatum was higher in the IDPN group than in the control group $(\mathrm{P}<0.05)$. Following treatment with NDG, the DAT content in the striatum was increased (IDPN+NDG group vs. IDPN+NS group, $\mathrm{P}<0.05)$. However, no significant difference was determined in the Hal group compared with the IDPN group (Fig. 3).

5-HT $T_{2 A} R$. There was a slight increase in the $5-\mathrm{HT}_{2 \mathrm{~A}} \mathrm{R}$ content in the striata of TS model rats, though this was not significant when compared with the content in control rats. There were no differences in the $5-\mathrm{HT}_{2 \mathrm{~A}} \mathrm{R}$ content in other brain regions

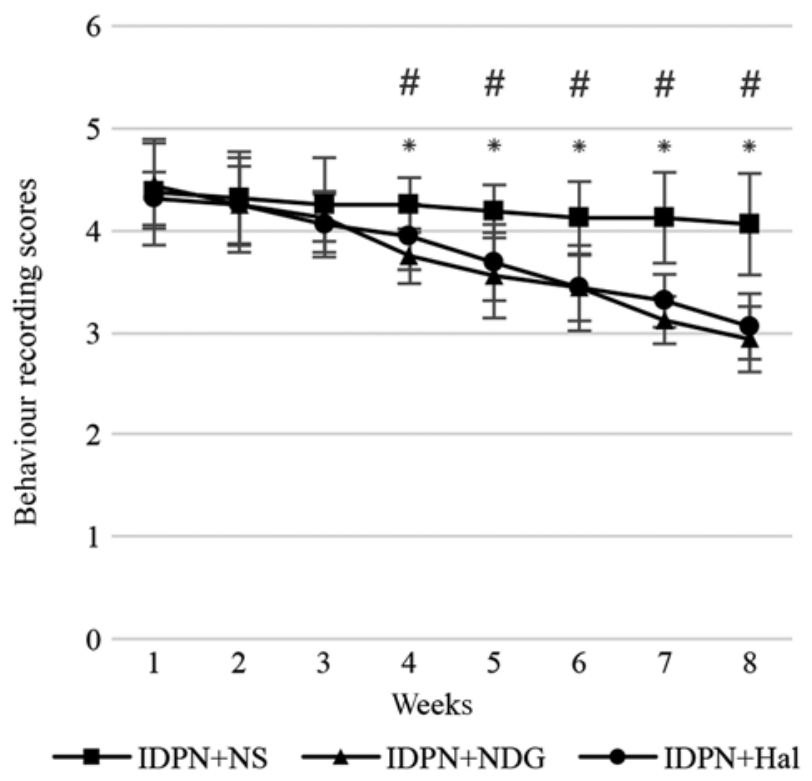

Figure 1. Evaluation of stereotypical behaviour scores of TS model rats in different groups during an 8 -week period. Data are given as the mean \pm SEM ( $n=6$ rats/group). The scores for the first 3 weeks revealed no differences between the groups $(\mathrm{P}>0.05)$. However, scores gradually decreased after 3 weeks in the NDG and Hal treatment groups, and there were no differences between these groups. ${ }^{\#} \mathrm{P}<0.05$ vs. IDPN+NDG; ${ }^{*} \mathrm{P}<0.05$ vs. IDPN+Hal. IDPN, 3,3'-Iminodipropionitrile; NDG, Ningdong granule; NS, normal saline; Hal, haloperidol.

among the groups. After 8 weeks of NDG administration, the uptake ratio of ${ }^{18} \mathrm{~F}$-altanserin was significantly increased in the striatum and cerebral cortex (IDPN+NDG group vs. IDPN+NS group, $\mathrm{P}<0.05)$. Meanwhile, Hal failed to stimulate any changes in $5-\mathrm{HT}_{2 \mathrm{~A}} \mathrm{R}$ content (IDPN+Hal group vs. IDPN+NS group, P>0.05; Fig. 4).

SERT. IDPN-treated rats exhibited no significant differences relative to the control group for all brain ROIs. Following treatment, NDG significantly reduced the SERT content in the nucleus accumbens, putamen, amygdaloid nucleus, striatum and cerebral cortex (IDPN+NDG group vs. IDPN+NS group, $\mathrm{P}<0.01)$. Hal reduced the SERT content in the nucleus accumbens and putamen (IDPN+Hal group vs. IDPN+NS group, 

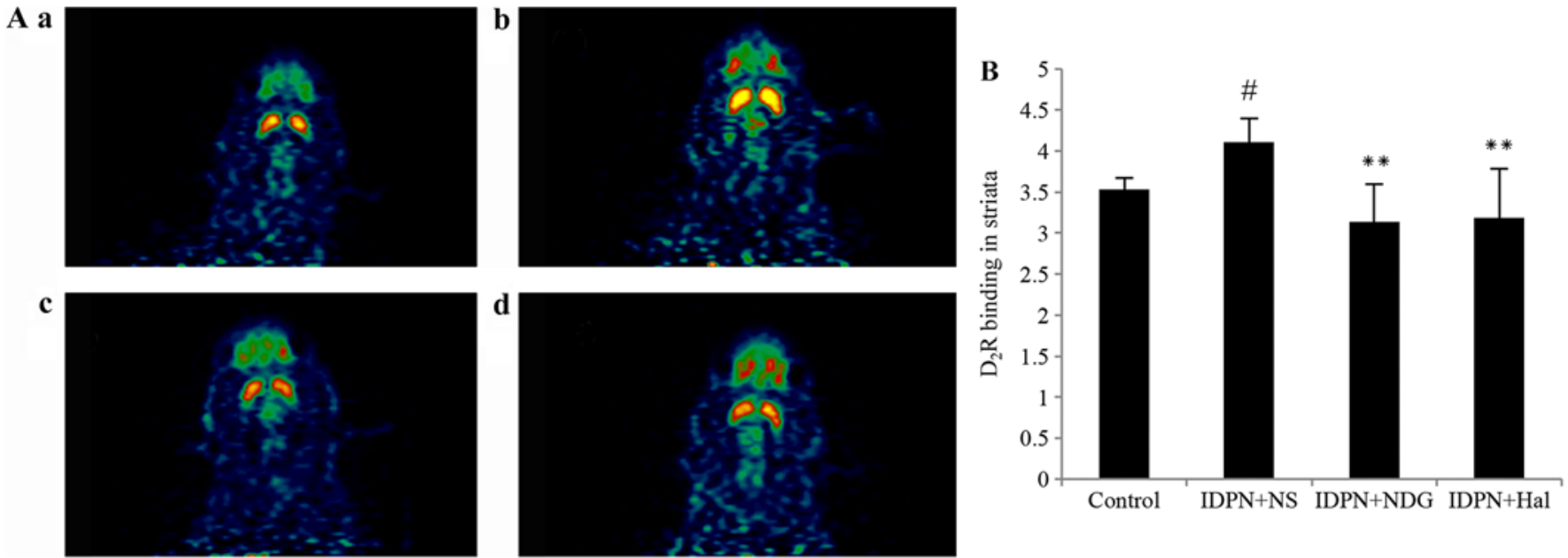

Figure 2. Micro-PET imaging and $\mathrm{D}_{2} \mathrm{R}$ binding in the bilateral striata. (A) Micro-PET imaging of bilateral striatum regions after an 8-week treatment. (Aa) Control group, (Ab) IDPN+NS group, (Ac) IDPN+NDG group and (Ad) IDPN+Hal group. Brighter areas reveal a higher uptake ratio in the brain (B) Binding potential of $\mathrm{D}_{2} \mathrm{R}$ in bilateral striata. Data are given as the mean $\pm \mathrm{SEM}\left(\mathrm{n}=6\right.$ rats/group). $\mathrm{P}<0.05$ vs. control; ${ }^{* *} \mathrm{P}<0.01$ vs. IDPN+NS. IDPN, 3,3'-Iminodipropionitrile; NDG, Ningdong granule; NS, normal saline; Hal, haloperidol; PET, positron emission tomography.
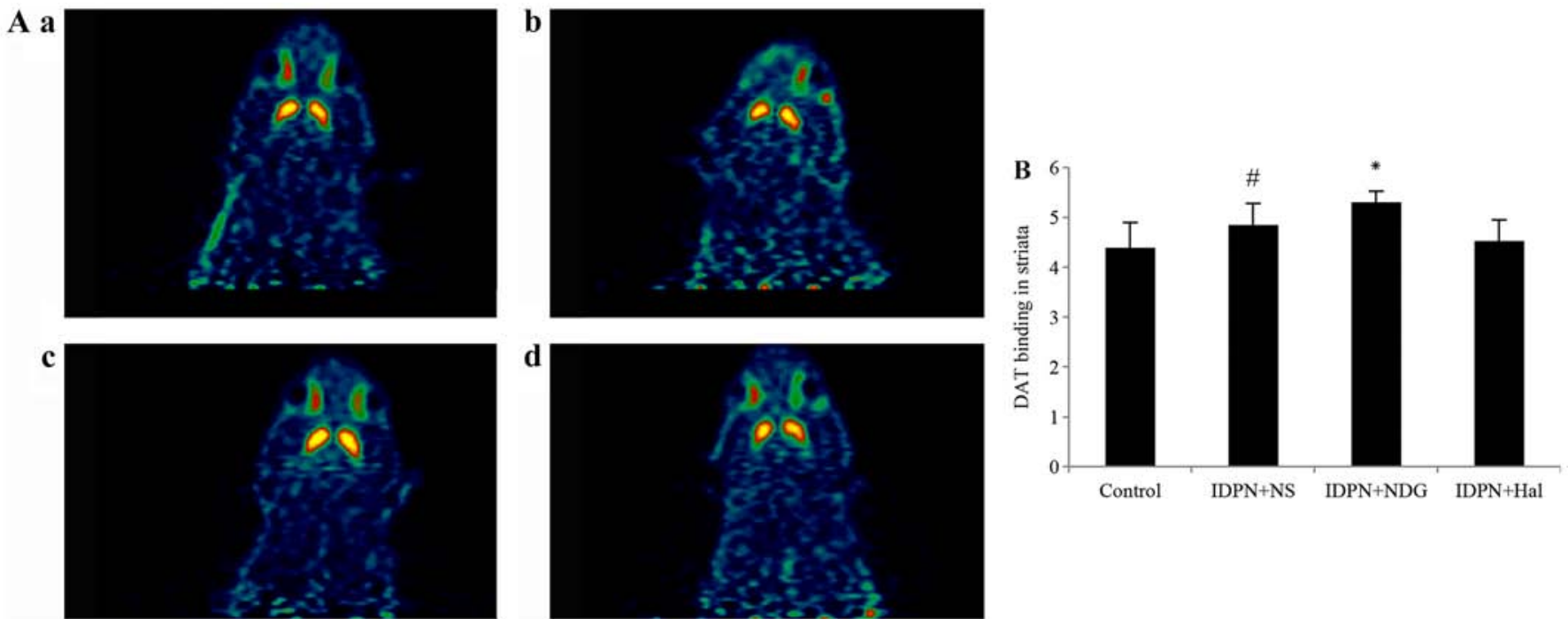

Figure 3. Micro-PET imaging and DAT binding in the bilateral striata. (A) Micro-PET imaging of bilateral striatum regions after an 8-week treatment. (Aa) Control group, (Ab) IDPN+NS group, (Ac) IDPN+NDG group and (Ad) IDPN+Hal group. Brighter areas reveal a higher uptake ratio in the brain. (B) Binding potential of DAT in bilateral striata. Data represent the mean $\pm \mathrm{SEM}$ ( $\mathrm{n}=6$ rats/group). ${ }^{*} \mathrm{P}<0.05$ vs. control; ${ }^{*} \mathrm{P}<0.05 \mathrm{vs}$. IDPN+NS. IDPN, 3,3'-Iminodipropionitrile; NDG, Ningdong granule; NS, normal saline; Hal, haloperidol; PET, positron emission tomography; DAT, dopamine transporter.

$\mathrm{P}<0.05)$. However, in other brain regions there were no significant differences between these groups (Fig. 5).

\section{Discussion}

Currently there is no model treatment for TS. Although the commonly used anti-tic drugs can alleviate the symptoms of tics, a series of side effects cause many patients to stop using these drugs. Hal is the drug most commonly used to suppress tics, attributable to its high efficacy as an antagonist of DA receptors; however, many patients stop using Hal due to its side effects that include sedation, electrocardiographic changes and extrapyramidal symptoms (23). NDG includes 10 Chinese herbal medicines, as described here, which are strictly based on the compatibility theory of TCM, and this formulation is specifically prepared for the treatment of TS. Previous clinical trials by our group have demonstrated the notable effects of NDG, with nearly one-half of the tested patients exhibiting positive outcomes; furthermore, NDG has also been demonstrated to ameliorate the symptoms of ADHD with few side effects [nausea (5.6\%), abdominal pain (5.6\%), increased appetite (13.9\%), difficulty in falling asleep (2.8\%), hypersomnia (16.7\%) and anxiety/nervousness (2.8\%)] (22). Meanwhile, our previous animal study demonstrated that NDG had dual ameliorative effects on the DA system (20). These initial results were confirmed in the current study with the use of high-resolution tomography in vivo that can provide more accurate parameters. Furthermore, the binding potential of DAT was measured in the current study. From the variation in DAT that was observed, it may be speculated that NDG 
A a

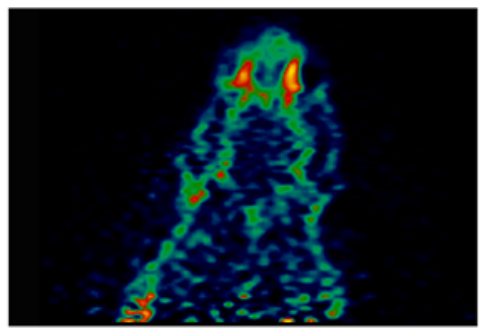

c

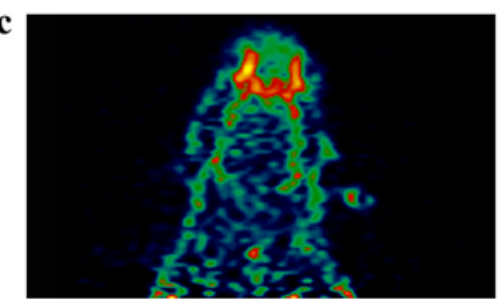

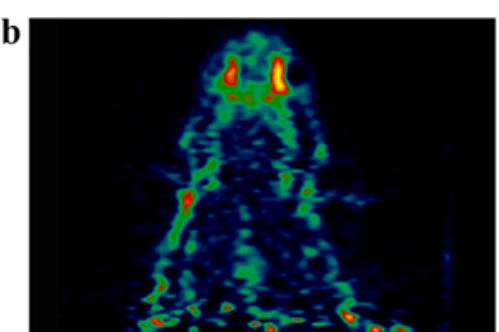

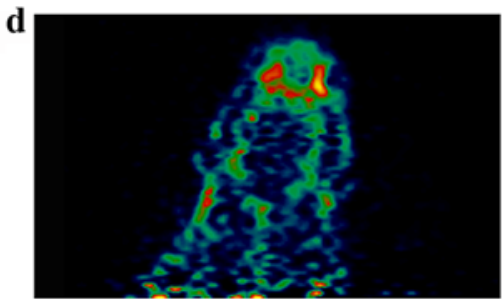

B

1.4

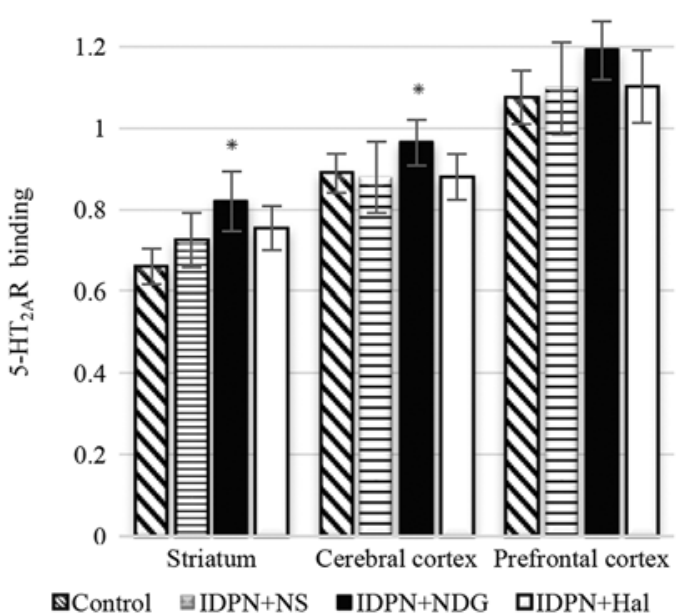

Figure 4. Micro-PET imaging and 5- $\mathrm{HT}_{2 \mathrm{~A}} \mathrm{R}$ binding in regions of interest. (A) Micro-PET imaging of brain regions after an 8-week treatment. (Aa) Control group, (Ab) IDPN+NS group, (Ac) IDPN+NDG group and (Ad) IDPN+Hal group. Brighter areas reveal a higher uptake ratio in the brain. (B) Binding potential of $5-\mathrm{HT}_{2 \mathrm{~A}} \mathrm{R}$ binding in regions of interest. Data represent the mean $\pm \mathrm{SEM}$ ( $\mathrm{n}=6$ rats/group). Only the NDG group exhibited a difference compared with the IDPN+NS group. ${ }^{*} \mathrm{P}<0.05$ vs. respective IDPN+NS group. IDPN, 3,3'-Iminodipropionitrile; NDG, Ningdong granule; NS, normal saline; Hal, haloperidol; PET, positron emission tomography; 5-HT, serotonin.
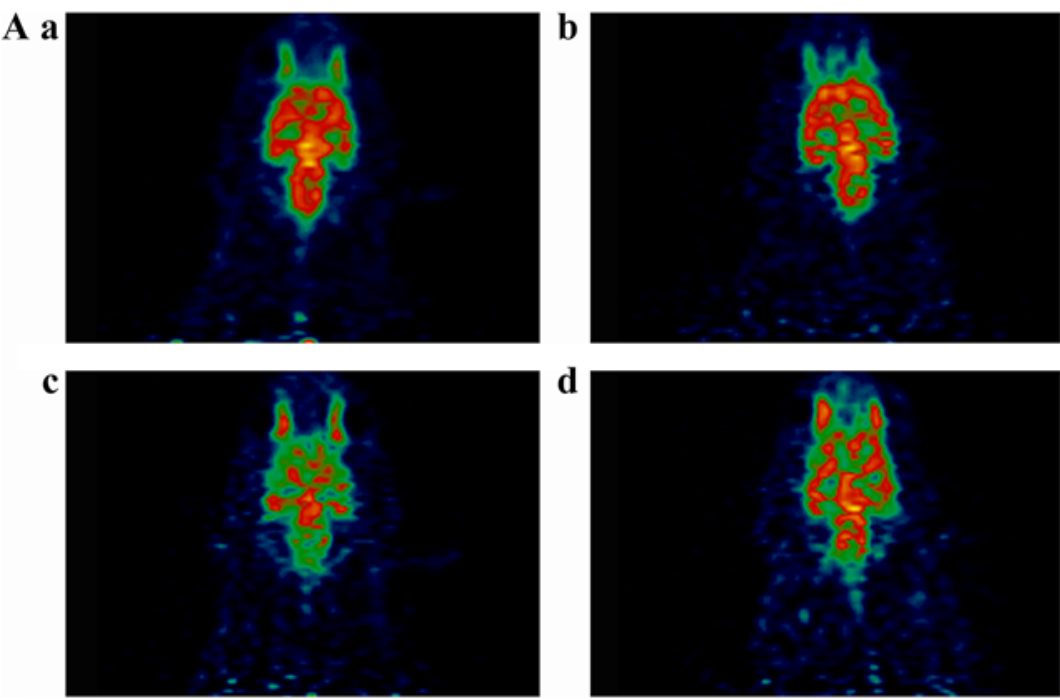

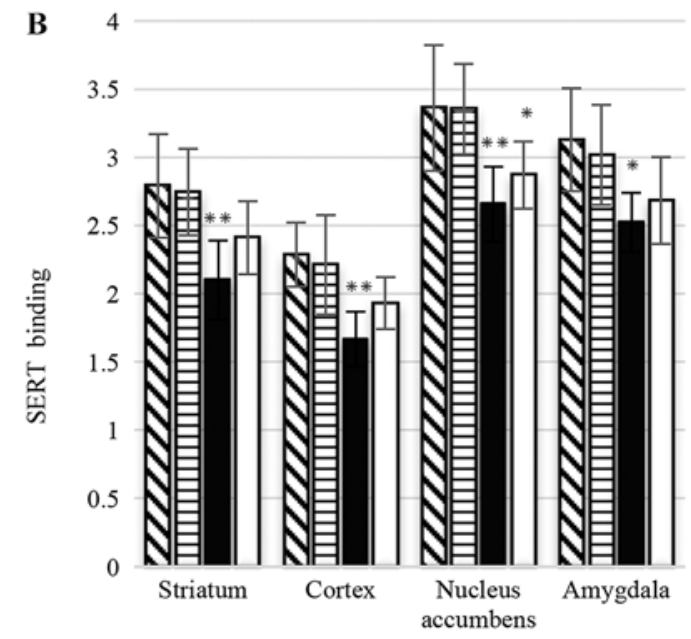

\$Control घIDPN+NS $\quad$ IDPN+NDG $\quad$ IIDPN+Hal

Figure 5. Micro-PET imaging and SERT binding in regions of interest. (A) Micro-PET imaging of brain regions after an 8-week treatment. (Aa) Control group, (Ab) IDPN+NS group, (Ac) IDPN+NDG group and (Ad) IDPN+Hal group. Brighter areas reveal a higher uptake ratio in the brain. (B) Binding potential of SERT in regions of interest. Data represent the mean \pm SEM ( $n=6$ rats/group). ${ }^{*} \mathrm{P}<0.05$ and ${ }^{* *} \mathrm{P}<0.01$ vs. respective IDPN+NS group. IDPN, 3,3'-Iminodipropionitrile; NDG, Ningdong granule; NS, normal saline; Hal, haloperidol; PET, positron emission tomography; SERT, serotonin transporters.

functions by upregulating DAT expression, thus weakening the hyperinnervation of DA fibers.

It has been suggested that there is a link between dopaminergic dysfunction and TS pathophysiology, but the results surrounding this assumption are heterogeneous. Increased as well as decreased receptor binding associated with TS, or no differences in receptor binding between patients with TS and control groups have been reported (24-26). Grace (27) proposed two components to describe the DA system: The phasic (spike-dependent) and tonic (homeostatic) components. In the model of tonic-phasic DA, increased DAT activity may lead to reduced tonic levels of DA, which could increase the levels of phasically released DA. Singer et al $(7,14)$ also reported increased DAT binding and proposed that increased DA release is a primary defect in TS. It may be speculated that DATs have both positive and negative effects on the regulation of DA. Normally, reuptake of the DA released into nerve terminals would be expected to reduce the concentration of DA in the synaptic cleft; however, the reduction of tonic DA levels may lead to phasic DA release. In the current study, IDPN exposure caused an increase in $\mathrm{D}_{2} \mathrm{R}$ and DAT binding compared with the levels in saline-treated control rats. Similar to the effects of Hal, NDG relieved the stereotypical symptoms of TS in rats and reversed the upregulation of striatal $\mathrm{D}_{2} \mathrm{R}$ density to normal levels. However, a significant increase in DAT binding was observed in the NDG-treated group, which was not observed in the Hal-treated group. 
5-HT is primarily produced in the brainstem by the raphe nuclei (28), which innervate virtually all regions of the central nervous system (29) and are responsible for regulating mood, eating behaviour, sleep and cognitive function. Serotonergic dysfunction was initially considered as the basis for OCD, a co-morbidity that is sensitive to selective 5-HT reuptake inhibitors. Experimental and clinical data have suggested that abnormalities in the serotonergic system are also associated with TS, and observations of interactions with the dopaminergic system have led to the hypothesis that 5-HT is associated with TS. Early studies revealed that $5-\mathrm{HT}_{2 \mathrm{~A}} \mathrm{R}$ binding is slightly increased in the occipital cortex and parietal cortex, while SERT binding potential (BP) was decreased (15). Lodge and Grace (30) documented that a deficit of 5-HT led to an increase in DA release; meanwhile, increased DA content could aggravate the symptoms of TS. Consistent with these findings, the present data indicated the presence of increased $5-\mathrm{HT}_{2 \mathrm{~A}} \mathrm{R}$ binding and decreased SERT binding in the rat model of TS. Treatment with NDG enhanced the increase in $5-\mathrm{HT}_{2 \mathrm{~A}} \mathrm{R}$ binding in the striatum and reduced the decrease in SERT BP in most brain ROIs. The increased $5-\mathrm{HT}_{2 \mathrm{~A}} \mathrm{R}$ binding and decreased SERT BP could be interpreted as a compensatory function to upregulate the content of 5-HT; increased 5-HT led to a decrease in DA release, which may alleviate the stereotypical abnormalities associated with TS.

In the DA system, NDG can decrease the content of DA by downregulating the binding of DA receptors and upregulating the content of DA transporters. In the 5-HT system, NDG can increase the content of 5-HT by upregulating the binding of 5-HT receptors and downregulating the content of 5-HT transporters, and indirectly decrease the content of DA. By interacting with these two neurotransmitter systems, NDG could effectively reduce the symptoms of TS.

Extensive experiments are being performed to study the relationship between tics and the dysfunction of the DA system, but few studies have assessed tics in the context of the dysfunction of serotonin metabolism. In the current study, the density, affinity and brain distribution of $\mathrm{D}_{2} \mathrm{R}, \mathrm{DAT}, 5-\mathrm{HT}_{2 \mathrm{~A}} \mathrm{R}$ and SERT were simultaneously measured in vivo, providing a more precise and greater insight into the pathogenesis of TS. Even so, the experimental procedure has certain limitations. Firstly, the diversity of individuals renders it difficult to reproduce the pathogenesis of human TS completely. Secondly, due to the limitation of time, the long-term efficacy of drugs cannot be observed. In the future, other monoamine neurotransmitters beyond DA and 5-HT, which might also participate in the pathophysiological course of TS, should be studied. In addition, it is important to try to develop a more effective and safe alternative treatment for TS.

The present study has demonstrated the effects of NDG treatment on stereotypical TS-related movement disorders in rats, involving neurotransmitter regulation. In conclusion, NDG may have promise as a safe and effective medication that can be used as an alternative therapy for TS. Additionally, the current findings suggest a model for an interaction between DA and 5-HT, where variation in the 5-HT system could be a compensatory mechanism to correct for dysfunction of the DA system. However, further understanding of the specific relationship between DA and 5-HT, as well as other neurotransmitters, is now required.

\section{Acknowledgements}

Not applicable.

\section{Funding}

The study was supported by the National Natural Science Foundation of China (grants no. 81273798 and no. 81503613), the Natural Science Foundation of Shandong province (grants no. ZR2012HM030 and no. BS2015YY030), the Development Project of Science and Technology of Traditional Chinese Medicine of Shandong Province (grant no. 2013ZDZK-085), the Development Projects of Shandong Province Science and Technology (grant no. 2011GSF11903) and the Postdoctoral Science Foundation of China (grant no. 2014M551924).

\section{Availability of data and materials}

The datasets used and/or analyzed during the present study are available from the corresponding author on reasonable request.

\section{Authors' contributions}

YW conducted the experiments and wrote the manuscript. AYL conceptualized and designed the study. All authors read and approved the final manuscript.

\section{Ethics approval and consent to participate}

All experimental procedures were performed in compliance with the relevant guidelines and regulations of the American Physiological Society, and the protocols were approved by the medical ethics committee of The Provincial Hospital Affiliated to Shandong University.

\section{Patient consent for publication}

Not applicable.

\section{Competing interests}

The authors declare that they have no competing interests.

\section{References}

1. Scharf JM, Miller LL, Gauvin CA, Alabiso J, Mathews CA and Ben-Shlomo Y: Population prevalence of Tourette syndrome: A systematic review and meta-analysis. Mov Disord 30: 221-228, 2015.

2. Houghton DC, Capriotti MR, Conelea CA and Woods DW: Sensory phenomena in tourette syndrome: Their role in symptom formation and treatment. Curr Dev Disord Rep 1: 245-251, 2014.

3. Pauls DL, Fernandez TV, Mathews CA, State MW and Scharf JM: The inheritance of tourette disorder: A review. J Obsessive Compuls Relat Disord 3: 380-385, 2014.

4. Paschou P, Yu D, Gerber G, Evans P, Tsetsos F, Davis LK, Karagiannidis I, Chaponis J, Gamazon E, Mueller-Vahl K, et al: Genetic association signal near NTN4 in Tourette syndrome. Ann Neurol 76: 310-315, 2014.

5. Prontera P, Napolioni V, Ottaviani V, Rogaia D, Fusco C, Augello B, Serino D, Parisi V, Bernardini L, Merla G, et al: DPP6 gene disruption in a family with Gilles de la Tourette syndrome. Neurogenetics 15: 237-242, 2014.

6. Leckman JF: Tourette's syndrome. Lancet 360: 1577-1586, 2002. 
7. Singer HS, Hahn IH and Moran TH: Abnormal dopamine uptake sites in postmortem striatum from patients with Tourette's syndrome. Ann Neurol 30: 558-562, 1991.

8. Wolf SS, Jones DW, Knable MB, Gorey JG, Lee KS, Hyde TM, Coppola R and Weinberger DR: Tourette syndrome: Prediction of phenotypic variation in monozygotic twins by caudate nucleus D2 receptor binding. Science 273: 1225-1227, 1996.

9. Minzer K, Lee O, Hong JJ and Singer HS: Increased prefrontal D2 protein in Tourette syndrome: A postmortem analysis of frontal cortex and striatum. J Neurol Sci 219: 55-61, 2004.

10. Haugbol S, Pinborg LH, Regeur L, Hansen ES, Bolwig TG, Nielsen FA, Svarer C, Skovgaard LT and Knudsen GM: Cerebral 5-HT2A receptor binding is increased in patients with Tourette's syndrome. Int J Neuropsychopharmacol 10: 245-252, 2007.

11. Martino D and Pringsheim TM: Tourette syndrome and other chronic tic disorders: An update on clinical management. Expert Rev Neurother 18: 125-137, 2018.

12. Leckman JF, Bloch MH, Smith ME, Larabi D and Hampson M Neurobiological substrates of Tourette's disorder. J Child Adolesc Psychopharmacol 20: 237-247, 2010.

13. George MS, Robertson MM, Costa DC, Trimble MR, Pilowsky L and Verhoeff NP: Dopamine receptor availability in Tourette's syndrome. Psychiatry Res 55: 193-203, 1994.

14. Singer HS, Szymanski S, Giuliano J, Yokoi F, Dogan AS, Brasic JR, Zhou Y, Grace AA and Wong DF: Elevated intrasynaptic dopamine release in Tourette's syndrome measured by PET. Am J Psychiatry 159: 1329-1336, 2002.

15. Wong DF, Brasic JR, Singer HS, Schretlen DJ, Kuwabara H, Zhou Y, Nandi A, Maris MA, Alexander M, Ye W, et al: Mechanisms of dopaminergic and serotonergic neurotransmission in Tourette syndrome: Clues from an in vivo neurochemistry study with PET. Neuropsychopharmacology 33: 1239-1251,2008.

16. Kakhi S and Soomro GM: Obsessive compulsive disorder in children and adolescents: Duration of maintenance drug treatment. BMJ Clin Evid 2015, 2015.

17. McDougle CJ,Epperson CN,Pelton GH, Wasylink S and Price LH A double-blind, placebo-controlled study of risperidone addition in serotonin reuptake inhibitor-refractory obsessive-compulsive disorder. Arch Gen Psychiatry 57: 794-801, 2000.

18. Cadet JL: The iminodipropionitrile (IDPN)-induced dyskinetic syndrome: behavioral and biochemical pharmacology. Neurosci Biobehav Rev 13: 39-45, 1989.
19. Ogawa N, Mizukawa K, Haba K and Sato H: Neurotransmitter and receptor alterations in the rat persistent dyskinesia model induced by iminodipropionitrile. Eur Neurol 30 (Suppl 1): S31-S40, 1990

20. Zhang F and Li A: Dual ameliorative effects of Ningdong granule on dopamine in rat models of Tourette's syndrome. Sci Rep 5: 7731, 2015.

21. Zhao L, Li AY, Lv H, Liu FY and Qi FH: Traditional Chinese medicine Ningdong granule: The beneficial effects in Tourette's disorder. J Int Med Res 38: 169-175, 2010.

22. Li JJ, Li ZW, Wang SZ, Qi FH, Zhao L, Lv H and Li AY: Ningdong granule: A complementary and alternative therapy in the treatment of attention deficit/hyperactivity disorder. Psychopharmacology (Berl) 216: 501-509, 2011.

23. Egolf A and Coffey BJ: Current pharmacotherapeutic approaches for the treatment of Tourette syndrome. Drugs Today (Barc) 50: 159-179, 2014.

24. Ernst M, Zametkin AJ, Jons PH, Matochik JA, Pascualvaca D and Cohen RM: High presynaptic dopaminergic activity in children with Tourette's disorder. J Am Acad Child Adolesc Psychiatry 38: 86-94, 1999.

25. Gilbert DL, Christian BT, Gelfand MJ, Shi B, Mantil J and Sallee FR: Altered mesolimbocortical and thalamic dopamine in Tourette syndrome. Neurology 67: 1695-1697, 2006.

26. Hwang WJ, Yao WJ, Fu YK and Yang AS: [99mTc] TRODAT-1/[123I]IBZM SPECT studies of the dopaminergic system in Tourette syndrome. Psychiatry Res 162: 159-166, 2008.

27. Grace AA: Phasic versus tonic dopamine release and the modulation of dopamine system responsivity: A hypothesis for the etiology of schizophrenia. Neuroscience 41: 1-24, 1991.

28. Dahlstroem A and Fuxe K: Evidence for the existence of monoamine-containing neurons in the central nervous System. I. demonstration of monoamines in the cell bodies of brain stem neurons. Acta Physiol Scand 232 (Suppl): S231-S255, 1964.

29. Butcher LL and Woolf NJ: Cholinergic and serotonergic systems in the brain and spinal cord: Anatomic organization, role in intercellular communication processes, and interactive mechanisms. Prog Brain Res 55: 1-40, 1982.

30. Lodge DJ and Grace AA: The hippocampus modulates dopamine neuron responsivity by regulating the intensity of phasic neuron activation. Neuropsychopharmacology 31: 1356-1361, 2006. 